\title{
Ritt's theorem and the Heins map in hyperbolic complex manifolds
}

\author{
Marco Abate* and Filippo Bracci* \\ October 2004
}

\begin{abstract}
Let $X$ be a Kobayashi hyperbolic complex manifold, and assume that $X$ does not contain compact complex submanifolds of positive dimension (e.g., $X$ Stein). We shall prove the following generalization of Ritt's theorem: every holomorphic self-map $f: X \rightarrow X$ such that $f(X)$ is relatively compact in $X$ has a unique fixed point $\tau(f) \in X$, which is attracting. Furthermore, we shall prove that $\tau(f)$ depends holomorphically on $f$ in a suitable sense, generalizing results by Heins, Joseph-Kwack and the second author.
\end{abstract}

2000 Mathematics Subject Classification: Primary 32H50. Secondary 32Q45, 37F99.

\section{Introduction}

The classical Wolff-Denjoy theorem (see, e.g., [A2, Theorem 1.3.9]) says that the sequence of iterates of a holomorphic self-map $f$ of the unit disk $\Delta \subset \mathbb{C}$, except when $f$ is an elliptic automorphism of $\Delta$ or the identity, converges uniformly on compact subsets to a point $\tau(f) \in \bar{\Delta}$, the Wolff point of $f$. Furthermore, if $\tau(f) \in \Delta$ then it is the unique fixed point of $f$; and if $\tau(f) \in \partial \Delta$ then it is still morally fixed, in the sense that $f(\zeta)$ tends to $\tau(f)$ when $\zeta$ tends to $\tau(f)$ non-tangentially.

In 1941 , Heins $[\mathrm{H}]$ proved that the map $\tau: \operatorname{Hol}(\Delta, \Delta) \backslash\{\mathrm{id}\} \rightarrow \bar{\Delta}$, associating to every elliptic automorphism its fixed point and to any other map its Wolff point, is continuous. More than half a century later, using the first author's version (see [A1]) of the Wolff-Denjoy theorem for strongly convex domains in $\mathbb{C}^{n}$, Joseph and Kwack [JK] extended Heins' result to strongly convex domains.

In 2002, the second author started investigating further regularity properties of the Heins map. If $D$ is a bounded domain in $\mathbb{C}^{n}$, then $\operatorname{Hol}(D, D)$ is a subset of the complex Banach space $H^{\infty}(D)^{n}$ of $n$-uples of bounded holomorphic functions defined on $D$; so one may ask whether the Heins map, when defined, is holomorphic on some suitable open subset of $\operatorname{Hol}(D, D)$. And indeed, in [B] the second author proved that, when $D$ is strongly convex, the Heins map is well-defined and holomorphic on $\operatorname{Hol}_{c}(D, D)$, the open subset of holomorphic self-maps of $D$ whose image is relatively compact in $D$.

The aim of this paper is to prove a similar result for the space $\operatorname{Hol}_{c}(X, X)$ of the holomorphic self-maps of a Kobayashi hyperbolic Stein manifold whose image is relatively compact in $X$. First of all, we shall generalize the classical Ritt's theorem, proving (Theorem 1.1) that every $f \in \operatorname{Hol}_{c}(X, X)$ admits a unique fixed point $\tau(f) \in X$; therefore the Heins map $f \mapsto \tau(f)$ is well-defined and continuous (Lemma 2.1).

To study further regularity properties of the Heins map, one apparently needs a complex structure on $\operatorname{Hol}_{c}(X, X)$. Unfortunately, we do not know whether such a structure exists in general; so we shall instead prove (Theorem 2.3) that the Heins map is holomorphic when restricted to any holomorphic family inside $\operatorname{Hol}_{c}(X, X)$, a fact equivalent to $\tau$ being holomorphic with respect to any sensible complex structure on $\operatorname{Hol}_{c}(X, X)$. For instance, we obtain (Corollary 2.4) that the Heins map is holomorphic on $\operatorname{Hol}_{c}(D, D)$ for any bounded domain $D$ in $\mathbb{C}^{n}$.

Finally, the first author would like to thank prof. Weiping Yin and the Capital Normal University of Beijing for the warm hospitality he enjoyed during his stay in China.

\section{Ritt's theorem}

Let $X$ be a complex manifold. We shall denote by $\operatorname{Hol}_{c}(X, X)$ the space of holomorphic self-maps $f: X \rightarrow X$ of $X$ such that $f(X)$ is relatively compact in $X$.

In 1920, Ritt $[\mathrm{R}]$ proved that if $X$ is a non-compact Riemann surface then every $f \in \operatorname{Hol}_{c}(X, X)$ has a unique fixed point $z_{0} \in X$. Furthermore, this fixed point is attractive in the sense that the sequence $\left\{f^{k}\right\}$ of iterates of $f$ converges, uniformly on compact subsets, to the constant map $z_{0}$. This theorem has been generalized to bounded domains in $\mathbb{C}^{n}$ by Wavre [W]; see also Hervé [He, p. 83]. Arguing as in [A2, Corollary 2.1.32] we shall now prove a far-reaching generalization of Ritt's theorem:

* Both authors have been partially supported by Progetto MIUR di Rilevante Interesse Nazionale Proprietà geometriche delle varietà reali e complesse. 
Theorem 1.1: Let $X$ be a hyperbolic manifold with no compact complex submanifolds of positive dimension. Then every $f \in \operatorname{Hol}_{c}(X, X)$ has a unique fixed point $z_{0} \in X$. Furthermore, the sequence of iterates of $f$ converges, uniformly on compact subsets, to the constant map $z_{0}$.

Proof: Since $X$ is hyperbolic, by [A3] the space $\operatorname{Hol}(X, X)$ of holomorphic self-maps of $X$ is relatively compact in the space $C^{0}\left(X, X^{*}\right)$ of continuous maps of $X$ into the one-point compactification $X^{*}=X \cup\{\infty\}$, endowed with the compact-open topology. If $f \in \operatorname{Hol}_{c}(X, X)$, this implies that the sequence of iterates of $f$ is relatively compact in $\operatorname{Hol}(X, X)$, because $f(X) \subset \subset X$.

Let then $\left\{f^{k_{\nu}}\right\}$ be a subsequence of $\left\{f^{k}\right\}$ converging to $h_{0} \in \operatorname{Hol}(X, X)$. We can also assume that $p_{\nu}=k_{\nu+1}-k_{\nu}$ and $q_{\nu}=p_{\nu}-k_{\nu}$ tend to $+\infty$ as $\nu \rightarrow+\infty$, and that there are $\rho_{0}, g_{0} \in \operatorname{Hol}(X, X)$ such that $f^{p_{\nu}} \rightarrow \rho_{0}$ and $f^{q_{\nu}} \rightarrow g_{0}$ in $\operatorname{Hol}(X, X)$. Then it is easy to see that

$$
h_{0} \circ \rho_{0}=h_{0}=\rho_{0} \circ h_{0} \quad \text { and } \quad g_{0} \circ h_{0}=\rho_{0}=h_{0} \circ g_{0},
$$

and so

$$
\rho_{0}^{2}=\rho_{0} \circ \rho_{0}=g_{0} \circ h_{0} \circ \rho_{0}=g_{0} \circ h_{0}=\rho_{0} .
$$

Thus $\rho_{0}$ is a holomorphic retraction, whose image is contained in the closure of $f(X)$, which is compact. This means (see Rossi [Ro] and Cartan $[\mathrm{C}]$ ) that $\rho_{0}(X)$ is a compact connected complex submanifold of $X$, i.e., a point $z_{0} \in X$. Therefore $\rho_{0} \equiv z_{0}$ and $z_{0}$ is a fixed point of $f$, since $f$ clearly commutes with $\rho_{0}$.

We are left to proving that $f^{k} \rightarrow z_{0}$, which implies in particular that $z_{0}$ is the only fixed point of $f$. Since $\left\{f^{k}\right\}$ is relatively compact in $\operatorname{Hol}(X, X)$, it suffices to show that $z_{0}$ is the unique limit point of any converging subsequence of $\left\{f^{k}\right\}$. So let $\left\{f^{k_{\mu}}\right\}$ be a subsequence converging toward a map $h \in \operatorname{Hol}(X, X)$. Arguing as before we find a holomorphic retraction $\rho \in \operatorname{Hol}(X, X)$ such that $h=\rho \circ h$. Furthermore, $\rho$ must again be constant; but since it is obtained as a limit of a subsequence of iterates of $f$, it must commute with $\rho_{0}$, and this is possible if and only if $\rho \equiv z_{0}$. But then $h=\rho \circ h \equiv z_{0}$ too, and we are done.

In particular this theorem holds for hyperbolic Stein manifolds, because a Stein manifold has no compact complex submanifolds of positive dimension.

Remark 1.1: If $f^{k} \rightarrow z_{0}$, then the spectral radius of $d f_{z_{0}}$ is strictly less than one. Indeed, if $d f_{z_{0}}$ had an eigenvalue $\lambda \in \mathbb{C}$ with $|\lambda| \geq 1$, then $d\left(f^{k}\right)_{z_{0}}$ would have $\lambda^{k}$ as eigenvalue, and $\lambda^{k} \nrightarrow \rightarrow 0$ whereas $d\left(f^{k}\right)_{z_{0}} \rightarrow O$.

\section{The Heins map}

Let $X$ be a hyperbolic manifold with no compact complex submanifolds of positive dimension. The Heins map of $X$ is the map $\tau: \operatorname{Hol}_{c}(X, X) \rightarrow X$ that associates to any $f \in \operatorname{Hol}_{c}(X, X)$ its unique fixed point $\tau(f) \in X$, whose existence is proved in Theorem 1.1.

The first observation is that the Heins map is continuous:

Lemma 2.1: Let $X$ be a hyperbolic manifold with no compact complex submanifolds of positive dimension. Then the Heins map $\tau: \operatorname{Hol}_{c}(X, X) \rightarrow X$ is continuous.

Proof: Let $\left\{f_{k}\right\} \subset \operatorname{Hol}_{c}(X, X)$ be a sequence converging toward a map $f \in \operatorname{Hol}_{c}(X, X)$; we must show that $\tau\left(f_{k}\right) \rightarrow \tau(f) \in X$.

First of all, we claim that the set $\left\{\tau\left(f_{k}\right)\right\}$ is relatively compact in $X$. Assume that this is not true; then, up to passing to a subsequence, we can assume that the sequence $\left\{\tau\left(f_{k}\right)\right\}$ eventually leaves any compact subset of $X$. Now, the set $f(X)$ is relatively compact in $X$; we can then find an open set $D$ in $X$ such that

$$
f(X) \subset \subset D \subset \subset X .
$$

We have $\tau\left(f_{k}\right) \notin \bar{D}$ eventually; therefore for $k$ large enough we can find $R_{k}>0$ such that

$$
\overline{B\left(\tau\left(f_{k}\right), R_{k}\right)} \cap D=\varnothing \quad \text { and } \quad \overline{B\left(\tau\left(f_{k}\right), R_{k}\right)} \cap \partial D \neq \varnothing,
$$

where $B(z, R)$ is the ball of center $z \in X$ and radius $R>0$ with respect to the Kobayashi distance of $X$. Choose $z_{k} \in \overline{B\left(\tau\left(f_{k}\right), R_{k}\right)} \cap \partial D$ for every $k$ large enough; since $\partial D$ is compact, up to a subsequence we can assume that $z_{k} \rightarrow z_{0} \in \partial D$. In particular, then, $f_{k}\left(z_{k}\right) \rightarrow f\left(z_{0}\right) \in f(X) \subset D$. But, on the other hand, we 
have $f_{k}\left(z_{k}\right) \in \overline{B\left(\tau\left(f_{k}\right), R_{k}\right)} \subset X \backslash D$ for all $k$ large enough, because $\tau\left(f_{k}\right)$ is fixed by $f_{k}$ and the Kobayashi distance is contracted by holomorphic maps; therefore $f\left(z_{0}\right) \in X \backslash D$, contradiction.

So $\left\{\tau\left(f_{k}\right)\right\}$ is relatively compact in $X$; to prove that $\tau\left(f_{k}\right) \rightarrow \tau(f)$ it suffices to show that $\tau(f)$ is the unique limit point of the sequence $\left\{\tau\left(f_{k}\right)\right\}$. But indeed if $\tau\left(f_{k_{\nu}}\right) \rightarrow x \in X$ we have

$$
f(x)=\lim _{\nu \rightarrow+\infty} f_{k_{\nu}}\left(\tau\left(f_{k_{\nu}}\right)\right)=\lim _{\nu \rightarrow+\infty} \tau\left(f_{k_{\nu}}\right)=x ;
$$

but $\tau(f)$ is the only fixed point of $f$, and we are done.

As stated in the introduction, our aim is to prove that the Heins map is holomorphic in a suitable sense. Since we do not know how to define a holomorphic structure on $\operatorname{Hol}_{c}(X, X)$ for general manifolds, we shall prove another result which is equivalent to the holomorphy of $\tau$ in any reasonable setting (see for instance Corollary 2.4 below). We shall need the following lemma:

Lemma 2.2: Let $P \subset \mathbb{C}^{n}$ be a polydisk centered in $p_{0} \in \mathbb{C}^{n}$, and $h: P \rightarrow \mathbb{C}^{n}$ a holomorphic map. Then there is a holomorphic map $A: P \rightarrow M(n, \mathbb{C})$, where $M(n, \mathbb{C})$ is the space of $n \times n$ complex matrices, satisfying the following properties:

(i) $h(z)-h\left(p_{0}\right)=A(z) \cdot\left(z-p_{0}\right)$ for all $z \in P$;

(ii) $A\left(p_{0}\right)=d h_{p_{0}}$;

(iii) for every polydisk $P_{1} \subset \subset P$ centered at $p_{0}$ there is a constant $C\left(P_{1}\right)>0$ such that $\|A\|_{P_{1}} \leq C\left(P_{1}\right)\|h\|_{P_{1}}$.

Proof: We can write

$$
h(z)-h\left(p_{0}\right)=\int_{0}^{1} \frac{\partial}{\partial t} h\left(z_{0}+t\left(z-p_{0}\right)\right) d t=\sum_{j=1}^{n}\left(z^{j}-p_{0}^{j}\right) \int_{0}^{1} \frac{\partial h}{\partial z^{j}}\left(z_{0}+t\left(z-p_{0}\right)\right) d t .
$$

Therefore taking

$$
A_{j}^{i}(z)=\int_{0}^{1} \frac{\partial h^{i}}{\partial z^{j}}\left(z_{0}+t\left(z-p_{0}\right)\right) d t
$$

the matrix $A=\left(A_{j}^{i}\right)$ clearly satisfies (i) and (ii), and (iii) follows from the Cauchy estimates.

Theorem 2.3: Let $X$ be a hyperbolic manifold with no compact complex submanifolds of positive dimension, $Y$ another complex manifold, and $F: Y \times X \rightarrow X$ a holomorphic map so that $f_{y}=F(y, \cdot) \in \operatorname{Hol}_{c}(X, X)$ for every $y \in Y$. Then the map $\tau_{F}: Y \rightarrow X$ given by $\tau_{F}(y)=\tau\left(f_{y}\right)$ is holomorphic. Furthermore, for every $y_{0} \in Y$ the differential of $\tau_{F}$ at $y_{0}$ is given by

$$
d\left(\tau_{F}\right)_{y_{0}}=\left(\mathrm{id}-d\left(f_{y_{0}}\right)_{\tau\left(f_{y_{0}}\right)}\right)^{-1} \circ d F_{\left(y_{0}, \tau\left(f_{y_{0}}\right)\right)}(\cdot, O) .
$$

Notice that, by Remark 1.1, id $-d\left(f_{y_{0}}\right)_{\tau\left(f_{y_{0}}\right)}$ is invertible.

Proof: Without loss of generality, we can assume that $Y$ is a ball $B^{m} \subset \mathbb{C}^{m}$ centered at $y_{0}$. Set $p_{0}=\tau\left(f_{y_{0}}\right)$, and let $P_{0} \subset X$ be the domain of a polydisk chart centered at $p_{0}$. Since $f_{y_{0}}\left(p_{0}\right)=p_{0}$, we can find a polydisk $P_{1} \subset \subset P_{0}$ centered at $p_{0}$ such that $f_{y_{0}}\left(P_{1}\right) \subset \subset P_{0}$. Furthermore, by Lemma 2.1 there is also a $\delta>0$ such that $\left\|y-y_{0}\right\|<\delta$ implies $\tau\left(f_{y}\right) \in P_{1}$ and $f_{y}\left(P_{1}\right) \subset \subset P_{0}$. This means that as soon as $y$ is close enough to $y_{0}$ we can work inside $P_{0}$ and assume, without loss of generality, that $X$ is contained in some $\mathbb{C}^{n}$.

Write $p_{y}=\tau\left(f_{y}\right) \in P_{1}$, and define $h_{y}: \overline{P_{1}} \rightarrow \mathbb{C}^{n}$ by $h_{y}=f_{y}-f_{y_{0}}$. We have

$$
p_{y}-p_{0}=f_{y_{0}}\left(p_{y}\right)-f_{y_{0}}\left(p_{0}\right)+h_{y}\left(p_{y}\right) ;
$$

therefore Lemma 2.2 applied to $f_{y_{0}}$ yields a matrix $A(y)$, depending continuously on $y$ by Lemma 2.1, such that

$$
p_{y}-p_{0}=A(y) \cdot\left(p_{y}-p_{0}\right)+h_{y}\left(p_{y}\right) .
$$

Since $A(y) \rightarrow d\left(f_{y_{0}}\right)_{p_{0}}$ as $y \rightarrow y_{0}$, for $y$ close to $y_{0}$ id $-A(y)$ is invertible, and so we can write

$$
p_{y}-p_{0}=(\mathrm{id}-A(y))^{-1} \cdot h_{y}\left(p_{y}\right) .
$$


Now, we have

$$
d F_{\left(y_{0}, \tau\left(f_{y_{0}}\right)\right)}(\cdot, O)=\operatorname{Jac}_{y}\left(f_{y}\left(p_{0}\right)\right)\left(y_{0}\right),
$$

where $\mathrm{Jac}_{y}$ is the Jacobian matrix computed with respect to the $y$ variables; in particular,

$$
h_{y}\left(p_{0}\right)-d F_{\left(y_{0}, \tau\left(f_{y_{0}}\right)\right)}\left(y-y_{0}, O\right)=o\left(\left\|y-y_{0}\right\|\right) .
$$

This means that to show that $\tau_{F}$ is holomorphic and $d \tau_{F}$ has the claimed expression it suffices to show that

$$
\lim _{y \rightarrow y_{0}} \frac{\left\|\tau_{F}(y)-\tau_{F}\left(y_{0}\right)-\left(\mathrm{id}-d\left(f_{y_{0}}\right)_{p_{0}}\right)^{-1} \cdot h_{y}\left(p_{0}\right)\right\|}{\left\|y-y_{0}\right\|}=0,
$$

which is equivalent to proving that

$$
\lim _{y \rightarrow y_{0}} \frac{\left\|\left(\mathrm{id}-d\left(f_{y_{0}}\right)_{p_{0}}\right) \cdot\left(p_{y}-p_{0}\right)-h_{y}\left(p_{0}\right)\right\|}{\left\|y-y_{0}\right\|}=0 .
$$

Now, (2.1) yields

$$
\begin{aligned}
\frac{\left\|\left(\mathrm{id}-d\left(f_{y_{0}}\right)_{p_{0}}\right) \cdot\left(p_{y}-p_{0}\right)-h_{y}\left(p_{0}\right)\right\|}{\left\|y-y_{0}\right\|} & =\frac{\left\|(\mathrm{id}-A(y)) \cdot\left(p_{y}-p_{0}\right)-h_{y}\left(p_{0}\right)+\left(A(y)-d\left(f_{y_{0}}\right)_{p_{0}}\right) \cdot\left(p_{y}-p_{0}\right)\right\|}{\left\|y-y_{0}\right\|} \\
& \leq \frac{\left\|h_{y}\left(p_{y}\right)-h_{y}\left(p_{0}\right)\right\|}{\left\|y-y_{0}\right\|}+\left\|A(y)-d\left(f_{y_{0}}\right)_{p_{0}}\right\| \frac{\left\|p_{y}-p_{0}\right\|}{\left\|y-y_{0}\right\|} .
\end{aligned}
$$

Since $h_{y}(z)$ is holomorphic both in $y$ and in $z$, we have

$$
h_{y}(z)-h_{y_{1}}\left(z_{1}\right)=O\left(\left\|y-y_{1}\right\|,\left\|z-z_{1}\right\|\right) ;
$$

in particular,

$$
h_{y}(z)=h_{y}(z)-h_{y_{0}}(z)=O\left(\left\|y-y_{0}\right\|\right)
$$

uniformly on $P_{1}$. So (2.1) implies that $p_{y}-p_{0}=O\left(\left\|y-y_{0}\right\|\right)$, and thus the second summand in (2.3) tends to zero as $y \rightarrow y_{0}$.

Finally, if we apply Lemma 2.2 to $h_{y}$ we get a matrix $B(y)$ and a constant $C>0$ such that

$$
\left\|h_{y}\left(p_{y}\right)-h_{y}\left(p_{0}\right)\right\| \leq\|B(y)\| \cdot\left\|p_{y}-p_{0}\right\| \leq C\left\|h_{y}\right\|_{P_{2}}\left\|p_{y}-p_{0}\right\|
$$

when $y$ is close enough to $y_{0}$, where $P_{2} \subset \subset P_{1}$ is a fixed polydisk centered at $p_{0}$. But then (2.4) yields

$$
\left\|h_{y}\left(p_{y}\right)-h_{y}\left(p_{0}\right)\right\|=O\left(\left\|y-y_{0}\right\|^{2}\right),
$$

and so (2.2) is proved.

If $X$ is a bounded domain in $\mathbb{C}^{n}$, then $\operatorname{Hol}_{c}(X, X)$ is an open subset of $H^{\infty}(X)^{n}$, the complex Banach space of $n$-uples of bounded holomorphic functions defined on $X$. Therefore in this case $\operatorname{Hol}_{c}(X, X)$ has a natural complex structure, and we obtain the following generalization of the main result in [B]:

Corollary 2.4: Let $D \subset \subset \mathbb{C}^{n}$ be a bounded domain. Then the Heins map $\tau: \operatorname{Hol}_{c}(D, D) \rightarrow D$ is holomorphic.

Proof: It follows from Theorem 2.3 and [FV, Theorem II.3.10]. 


\section{References}

[A1] M. Abate: Horospheres and iterates of holomorphic maps. Math. Z. 198 (1988), 225-238.

[A2] M. Abate: Iteration theory of holomorphic maps on taut manifolds. Mediterranean Press, Cosenza, 1989.

[A3] M. Abate: A characterization of hyperbolic manifolds. Proc. Amer. Math. Soc. 117 (1993), $789-793$.

[B] F. Bracci: Holomorphic dependence of the Heins map. Complex Variables Theory and Appl. 47 (2002), 1115-1119.

[C] H. Cartan: Sur les rétractions d'une variété. C.R. Acad. Sci. Paris 303 (1986), 715-716.

[FV] T. Franzoni, E. Vesentini: Holomorphic maps and invariant distances. North Holland, Amsterdam, 1980.

[H] M.H. Heins: On the iteration of functions which are analytic and single-valued in a given multiplyconnected region. Amer. J. Math. 63 (1941), 461-480.

[He] M. Hervé: Several complex variables. Local theory. Oxford University Press, London, 1963.

[JK] J.E. Joseph, M.H. Kwack: A generalization of a theorem of Heins. Proc. Amer. Math. Soc. 128 (2000), 1697-1701.

[R] J.F. Ritt: On the conformal mapping of a region into a part of itself. Ann. of Math. 22 (1920), $157-160$.

[Ro] H. Rossi: Vector fields on analytic spaces. Ann. of Math. 78 (1963), 455-467.

[W] R. Wavre: Sur la réduction des domaines par une substitution à $m$ variables complexes et l'existence d'un seul point invariant. Enseign. Math. 25 (1926), 218-234.

Marco Abate

Dipartimento di Matematica, Università di Pisa

Largo Pontecorvo 5, 56127 Pisa, Italy

E-mail: abate@dm.unipi.it

Filippo Bracci

Dipartimento di Matematica, Università di Roma "Tor Vergata"

Via della Ricerca Scientifica, 00133 Roma, Italy

E-mail: fbracci@mat.uniroma2.it 